\title{
Effects of Double Bond Configuration on Lecithin Synthesis ${ }^{1}$
}

\author{
W. E. M. LANDS, Department of Biological Chemistry, The University of Michigan, Ann Arbor, Michigan
}

\begin{abstract}
Stearyl-CoA, oleyl-CoA and elaidyl-CoA were prepared and tested as substances in synthesizing lecithin from 1- and 2-acyglycerol-3-phosphorylcholine. The rates of acyltransfer were followed by a continuous spectrophotometric assay. The enzyme (s) used to catalyze esterification at the 2-position did not discriminate appreciably between the $c i s$ and trans isomers of octadecenoate and transferred them more rapidly than the saturated acid, octadecanoate. However, the enzyme(s) which acted at the 1-position discriminated markedly between the geometric isomers and rapidly transferred to trans-octadecenoate and octadecanoate.
\end{abstract}

\section{Introduction}

In 1935, Sinclatr described the use of trans- octa1 decenoate in elaidinized olive oil as a metabolic tracer to follow some general aspects of fat metabolism (1). He showed that elaidate rapidly entered the fats and phospholipids of liver and that it was later rapidly displaced by the naturally-occurring acids when the diet was restored to normal. Recent reports by Coots (2) and Ono and Fredrickson (3) indicate again that elaidate is not much different from other fatty acids in its general metabolic properties such as intestinal absorption and production of respiratory $\mathrm{CO}_{2}$. Sinclair noted, however, that the per cent of solid acids in the phospholipid remained constant and coneluded that elaidate was primarily displacing the normal saturated acids rather than the oleate. Although elaidate enters most of the glycerolipid fractions of a wide variety of tissues, Sinclair reported (4) that the trans isomer is partly excluded from the brain lipids and almost completely excluded from phospholipids of the testes of white rats.

Acyl-CoA : phospholipid acyltransferases can be quite selective in terms of chain length and degree of unsaturation (5). Studies with this enzymatic system suggest that each individual acid, laurate, palmitate, stearate, oleate, linoleate and perhaps even elaidate, will have its own characteristic metabolic distribution and fate. The experiments reported in this paper were designed to see if the acyltransferase enzymes could discriminate between the geometrical isomers of octadecenoate and whether the more linear trans isomer behaved like a saturated fatty acid.

\section{Experimental Procedure}

The two isomers of acyl-GPC were prepared by the reactions indicated below.

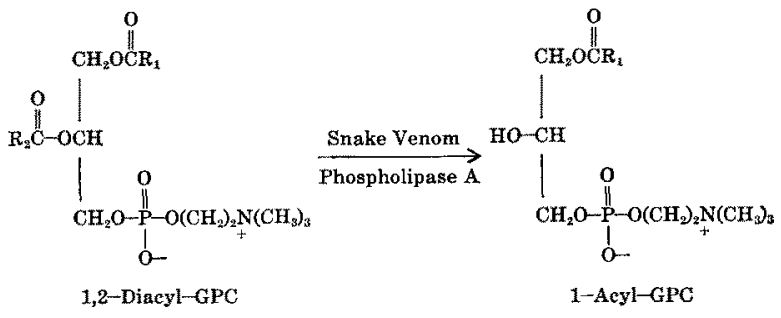

${ }^{1}$ AOCS Bond Award paper. Presented at the AOOS Meeting, Ohicago, 1964 .
Purified diacyl-GPC (1,000 $\mu$ moles) from egg yolks (6) was dissolved in $25 \mathrm{ml}$ of diethylether and treated with $0.25 \mathrm{ml}$ of $C$. adamanteus venom $(2.5 \mathrm{mg})$ in Tris buffer ( $\mathrm{pH} 7.4,0.1 \mathrm{M}$ ) containing $10^{-3} \mathrm{M} \mathrm{CaCl}_{2}$. The mixture was shaken vigorously and allowed to react for $3 \mathrm{hr}$. Acyl-GPC precipitated from solution and was removed by centrifugation and washed thoroughly with dry ether to remove free acids and any unreacted diacyl-GPC. The precipitate was dissolved in warm ethanol to denature the enzymes, the solution evaporated to dryness, and the lipid was dissolved in chloroform :methanol $(2: 1)$.

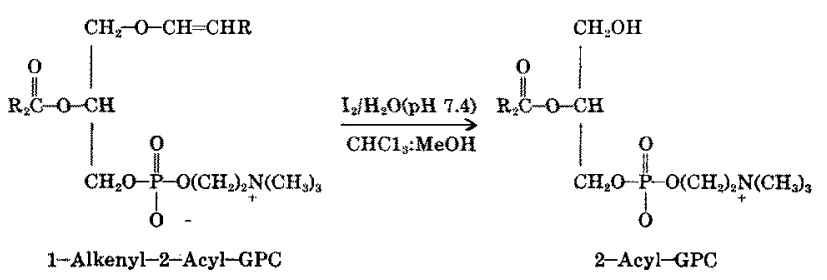

The 2-acyl isomer was prepared by treating 25 $\mu$ moles of beef heart cholinephosphoglycerides ( $40 \%$ plasmalogen) in $2 \mathrm{ml}$ of chloroform :methanol (2:1) with $1 \mathrm{ml}$ of $0.1 M$ Tris buffer $(\mathrm{pH} 7.4)$ and $0.7 \mathrm{ml}$ (14 $\mu$ moles) of aqueous $\mathrm{I}_{2}$. After $10 \mathrm{~min}, 3 \mathrm{ml}$ of ethanol were added, the excess $\mathrm{I}_{2}$ was reacted with $\mathrm{Na}_{2} \mathrm{~S}_{2} \mathrm{O}_{3}$, and the chloroform solution was washed twice with water to remove salts. The lipid extract was evaporated to dryness, treated twice with acetone to remove organic and inorganic iodides and then dispersed in $10 \mathrm{ml}$ of water.

Acyl-CoA derivatives were prepared from the acid chlorides by the method of Seubert (7) and stored as dilute solutions $\left(2 \times 10^{-3} M\right)$ at $-10 \mathrm{C}$. The enzyme preparations were made from tissue homogenates in $0.25 \mathrm{M}$ sucrose.

The rate and extent of the acyltransferase reaction was followed continuously using an internal colorimetric reagent for the liberated sulfhydryl group of CoASH. This reagent, 5,5'-dithio-bis-(2-nitrobenzoate) (DTNB), was described by Ellman (8). A representative enzymatic reaction mixture contained $1.0 \mu$ mole of DTNB, $100 \mathrm{~m} \mu$ moles of acyl-GPC, $50 \mathrm{~m} \mu$ moles of acyl-CoA, and $80 \mu$ moles of Tris buffer ( $\mathrm{pH} 7.4$ ) in a final volume of $1 \mathrm{ml}$. The increase in absorbancy at $412 \mathrm{~m} \mu$ is an indication of the increase in the CoASH formed from the thiol ester as shown below.

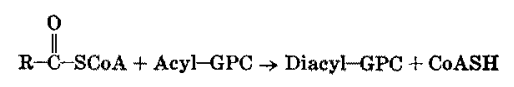

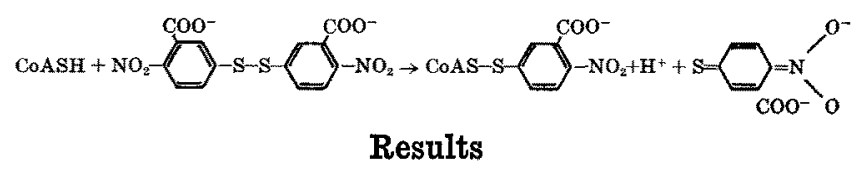

When 2-acyl-GPC was the phospholipid acceptor, elaidate and stearate were rapidly transferred from their $\mathrm{CoA}$ derivatives to form diacyl-GPC (Fig. 1). The bottom curve shows the rate of increase of $a b$ sorbancy when no lipid was added. The liberated 


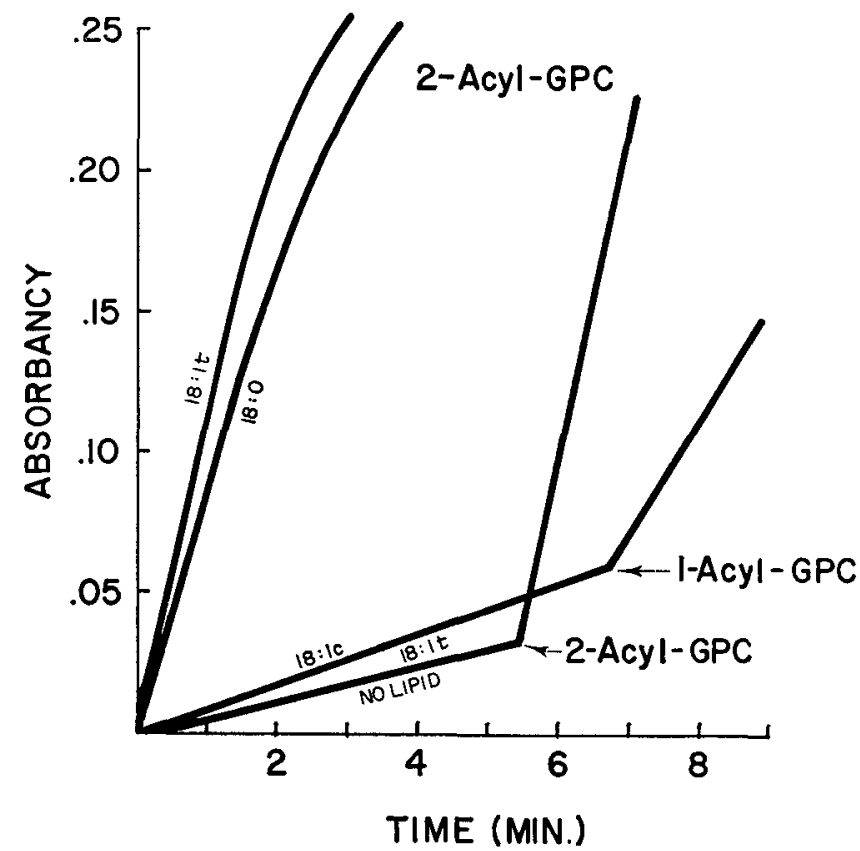

FIG. 1. Relative acyltransferase rates with stearate $(18: 0)$, elaidate $(18: 1 \mathrm{t})$ and oleate $(18: 1 \mathrm{e})$.

mercaptan is due to a slight amount of hydrolysis. When 2-acyl-GPC was added after $5 \mathrm{~min}$, the rate was identical to that when all components were present initially. The cis-octadecenoate reacted not much faster than the control level seen for hydrolysis, but when the 1-acyl-GPC isomer was added, the acyl transfer proceeded at a good rate. The rates of the acyl transfers with stearate, elaidate and oleate and the two positional isomers of acyl-GPC using pig liver microsomes are shown in Table I. The cis-isomer was esterified more rapidly at the 2-position and the trans-isomer at the 1-position. A similar specificity for the geometrical isomers was shown by rat liver mitochondria (Table II). These experiments also show the effect of chain length as well as unsaturation on the acyltransfer rates. The shorter saturated acids behave more like the unsaturated acids than the longchain saturated ones do.

\section{Discussion}

The role of acyltransferases in lecithin formation is indicated below. Lecithin formed by the pathway described by Kennedy (9) would be expected to have a pattern of acids like that in the diglyceride percursor. This pattern could then be arranged by the

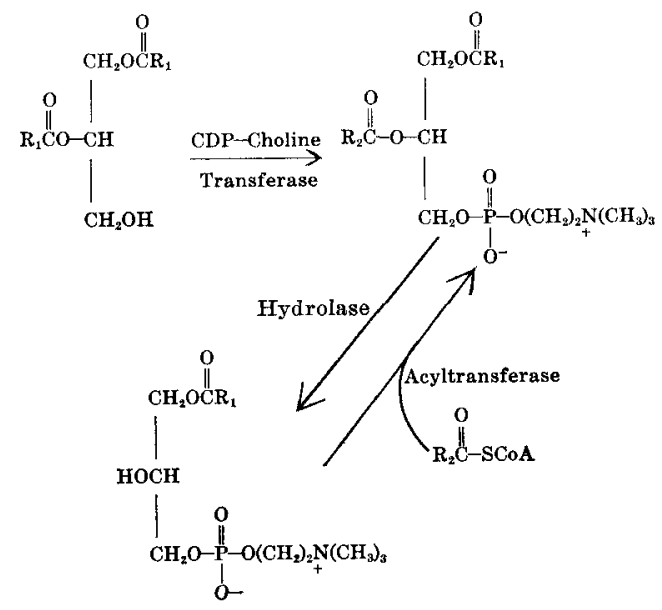

TABLE T

Acyl Transfer Catalyzed by Pig Liver Microsomesa

\begin{tabular}{llcc}
\hline & Acyl-CoA & 1-Acyl-GPO & 2-Acyl-GPC \\
\hline & $18: 0$ & 3.0 & 28.5 \\
Exp. 1 & $18: 1(\mathrm{t})$ & 15.5 & 42.0 \\
Exp. 2 & $18: I(\mathrm{c})$ & 13.5 & 1.0 \\
& $18: 1(\mathrm{t})$ & 20.5 & $\mathbf{4 4 . 5}$ \\
\hline
\end{tabular}

a'The reaction mixtures were prepared as described in the Exprimental section and the results are given in terms of mamoles $/ \mathrm{min} / \mathrm{mg}$

action of a hydrolase and an acyltransferase. Thus in the example shown, a new acid is esterified at the 2-position and the fatty acid composition at that position could eventually reflect the specificity of that acyltransferase and the supply of available acyl-CoA derivatives.

There have been a number of generalizations regarding the role that saturated and unsaturated acids may play in affecting the physiological state of an animal, but we have yet to find the specific metabolic steps by which this effect is exerted. Results with the acyltransferases have paralleled those found earlier in dietary experiments with intact animals in showing that some saturated acids of shorter chain length actually behave more like the unsaturated long-chain acids. In addition the dienoic acid, linoleate, is incorporated more selectively into the 2-position than is the monoenoic acid, oleate, and elaidate is incorporated more rapidly into the 1-position than is oleate. Thus the general classification of saturated and unsaturated acid is not precise enough to indicate the metabolic fate of an acid, and we should expect that no single fatty acid will be a suitable general representative for all the other acids. Apparently each fatty acid has its own distribution pattern within the phospholipid that can be controlled in part by selective acyltransferases.

The enzyme (s) used in these experiments to catalyze esterification at the 2-position did not discriminate appreciably between the cis and trans isomers of octadecenoate. However, the enzymes which acted at the 1-position diseriminated markedly between the geometric isomers, and treated the trans-octadecenoate almost as if it were the more linear, saturated acid, octadecanoate. The experiments of Ono and Fredrickson (3) were designed to measure possible small differences in several metabolic systems characterized by rapid turnover, but no appreciable differences were found. In contrast, the results in this paper show that the acyltransferase reaction at the primary hydroxyl of 2-acyl-GPC is markedly sensitive to the configuration of the double bond. Raulin et al. (10) showed also that the trans isomer was principally incorporated into the primary positions of depot triglycerides of rats that were fed a diet of elaidinized peanut oil for two to seven months. As our techniques improve for studying specific acyltransferases from various tissues, it may be possible to predict by fast enzymatic experiments the results that are

TABLE II

Acyl Transfer Catalyzed by Rat Liver Mitochondria*

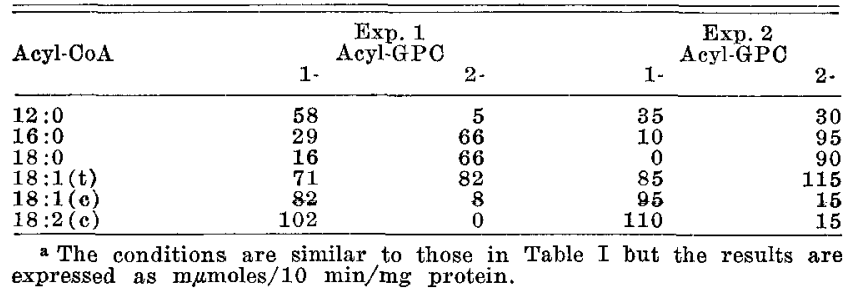


eventually obtained from long-term [e.g., 84 days (11)] dietary studies with elaidate and other unusual fatty acids. In particular, some enzymatic basis may be found to indicate why the "cholesterol coefficient" of the trans monoenoic acids $(+2.1)$ is so similar to that for the saturated acids $(+2.7)$ whereas that for the cis isomers is essentially zero (12). At the present we can see an interesting similarity in the acyltransferase specificities at the 1-position of 2-acyl-GPC and these coefficients.

\section{ACKNOWLEDGMENTS}

Technical assistance was provided by P. Hart Coleman. The work was supported in part by a grant (AM-05310) from the U.S. Publie Health Service.
REFERENOES

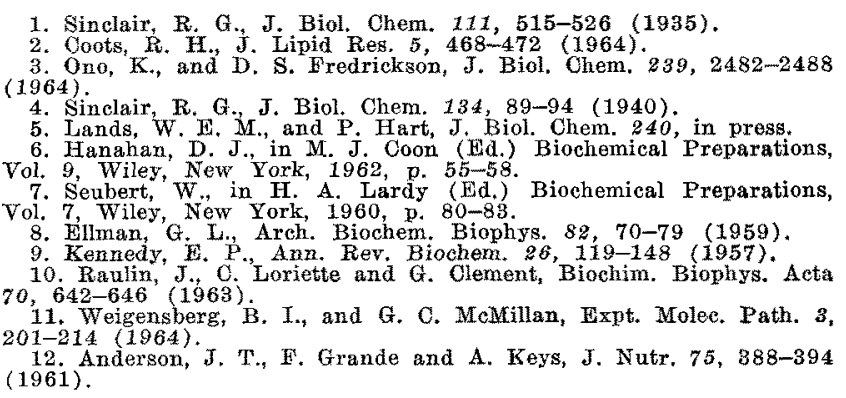

[Received January 4, 1965--Aceepted February 24, 1965]

\title{
Assay of Aflatoxin in Peanuts and Peanut Products
} Using Acetone-Hexane-Water for Extraction ${ }^{1}$

\author{
J. A. ROBERTSON, JR., LOUISE S. LEE, ALVA F. CUCULLU and L. A. GOLDBLATT, ${ }^{2}$ \\ Southern Regional Research Laboratory, New Orleans, Louisiana
}

\begin{abstract}
A quantitative method is described for the assay of aflatoxin in peanut products. The procedure involves extraction of aflatoxin from the sample with a homogeneous acetone-hexane-water solvent mixture followed by purification of the extract by phasic extraction of the aflatoxin with aqueous sodium chloride and then with chloroform. The purified chloroform extract is analyzed by thin-layer chromatography by comparison of the intensity of fluorescence of any aflatoxin with. the intensity of a known standard. The aflatoxin analyses of peanuts were found to be very variable due to sampling, and this variability has been greatly reduced by finely grinding and thoroughly mixing $2 \mathrm{~kg}$ of the sample before removal of an aliquot for assay. The method is sensitive to approximately 2 parts per billion.
\end{abstract}

\section{Introduction}

$A^{n}$ FLATOXINS ARE HIGHLY toxic metabolites initially found to be produced by species of the common mold Aspergillus flavus (1) and later found to be produced by other mold species $(2,3)$. The aflatoxins were first found in a peanut meal which caused the death of a large number of farm animals (4). The toxins are not limited to peanut products but also have been found associated with the toxicity caused by other agricultural commodities $(4,5)$.

Crude aflatoxin is a mixture of several fluorescent components, four of which have been isolated in a pure state and shown to be toxic in varying degrees to ducklings $(6)$. These four components were designated aflatoxin $B_{1}, B_{2}, G_{1}$ and $G_{2}$ because of their origin as metabolites of Aspergillus flavus and from the blue and green fluorescence they exhibit when exposed to ultraviolet (UV) radiation. The structure of aflatoxin $B_{1}$ and $G_{1}$ has been established by Asao et al. (17). Aflatoxin $B_{2}$ and $G_{2}$ are dihydro derivatives of the respective $B_{1}$ and $G_{1}(6,8,9)$.

\footnotetext{
1 Honorable mention, Bond Award competition. Presented at AOCS Meeting, Chicago, Illinois, Oetober, 1964

2 So. Reg. Res. Lab., New Orleans, Louisiana, one of the laboratories of the So. Utiliz. Res. and Dev. Div., ARS, USDA.
}

The aflatoxins are soluble in solvents such as methanol, and this solubility forms the basis for most of the present methods of assay (10-14). Some of these methods are long and time-consuming and lack sensitivity due to quenching of fluorescence by impurities.

Until quite recently all the procedures used for the determination of aflatoxin in peanut products called for initial separation of the aflatoxin by prolonged Soxhlet extraction. For this procedure a single solvent or a constant boiling mixture of solvents possesses obvious advantages. In this laboratory an azeotrope of acetone, hexane and water has been under investigation as a solvent for removal of lipids and gossypol from cottonseed for several years (15). The use of this azeotrope was investigated, and it was found to be an excellent extractant for aflatoxin from peanut products with the added advantage that it does not extract as much extraneous material as does methanol. Thus, equal amounts of aflatoxin were found in a commercial peanut meal when the aflatoxin was removed by $6 \mathrm{hr}$ of Soxhlet extraction with methanol or with the acetone-hexane-water azeotrope. However, the residue obtained after evaporation of the methanolic extract from $100 \mathrm{~g}$ of commercial peanut meal amounted to $11.55 \mathrm{~g}$, whereas that obtained after evaporation of the azeotrope amounted to only $3.13 \mathrm{~g}$. Accordingly, the subsequent purification of the extract required for assay for aflatoxin by thin-layer chromatography (TLC) is much simpler if the azeotrope is used as the extractant.

Recently, it has been found in assaying for aflatoxin that equally satisfactory results can be obtained in much shorter extraction time by equilibrium extraction using a Waring Blendor or a shaker (16). The azeotrope is not completely miscible at room temp, and a small lower layer relatively richer in acetone and water separates out, thus making it somewhat inconvenient to handle in the laboratory. Since the constant boiling temp characteristic of an azeotrope is not important in equilibrium extraction, the utility, as an extractant for aflatoxin, of homogeneous mixtures of the solvents was investigated. A mixture of acetone, hexane and water in the proportion of $5: 48.5: 1.5(\mathrm{v} / \mathrm{v})$ was found to be homogeneous and to 